\title{
Novel Statistical Arguments and Stability Coefficient Methods in Dynamic Reducts
}

\author{
Wang Jiayang and Khaled Omar Salem AL-wesabi
}

\begin{abstract}
The paper discusses the methods used to rough set theory knowledge to discovery from decision tables. From this ways dynamic reduct. Discovery relative static and dynamic reducts, discovery frequent sets, discovery decision rules and calculate stability coefficients for objects from a decision table. The most important and matchless feature of dynamic reduct is ability to calculate stability coefficients for every object from decision table using different methods. In this study we present some statistical arguments showing the introduced stability coefficient of dynamic reducts are proper measures of their quality.
\end{abstract}

Index Terms-Dynamic reduct, Decision table, Rough set, Stability coefficients.

\section{INTRODUCTION}

Some methods for extracting laws from data based on rough set approach and Boolean reasoning are presented in this paper. Standard rough set methods are not always sufficient for extracting laws from decision tables [1]. Because is these methods are not taking into account the fact that part of the reduct set is chaotic i.e. is not stable in randomly chosen samples of a given decision table. A method for selection of feature (attribute) sets relevant for extracting laws from data is proposed. These sets of attributes are called dynamic reducts. The most important and matchless feature of dynamic reduct is ability to calculate stability coefficients for every object from decision table using different methods.

In this study will be displayed compute dynamic reducts and the decision rules on the basis of dynamic reducts. The goal from the study is to resent a method for extracting laws from experimental data. Problems related to extracting laws from experimental data are intensively studied. Some statistical arguments showing that dynamic reducts offer a good tool for extracting laws from decision table are given.

The results are suggesting that methods using dynamic reducts can be treated as a promising tool for extracting laws from experimental data sets.

The paper is organized as follows. Section 2. Stability coefficients calculation. Section 3. Definitions of dynamic reduct. Section 4 gives the Definition of generalized dynamic reduction, Section 5 Statistical arguments showing the introduced stability coefficient of dynamic reducts, In Section 6. Implementation stability coefficient, In Section 7.

Manuscript received September 12, 2012; revised October 12, 2012. The project supported by National Natural Science Foundation of China (No.61173052) and supported by Hunan Provincial Natural Science Foundation of China (No.11JJ5040).

The authors are with School of Information Science and Technology, Central South University, Changsha, China (e-mail address: csuwjy@mail.csu.edu.cn)
Introduces experimenttal result and discussion, concluding remarks are provided in section 8 .

\section{Stability Coefficients Calculation}

Stability coefficient of object is a measure of its similarity with respect to the rest of the objects in the considered information system [2]. There is no shared method to define the relation of similarity, so it is exactly connected with the implemented way.

All stability coefficients implemented in dynamic reduct are normalized to range $[0,1]$. Value 0 represents the least level of stability of a given object, while value 1 is the maximum representing perfect relationship of similarity to other objects in the decision table [3],[4]. This normalization is very useful especially when we want to compare results returned by different algorithms.

This module allows in calculation of stability coefficients with the use of the methods that is discussed in the following section

\section{DYNAMIC REDUCT}

Reducts generated from an information system are sensitive to changes in the system. This can be seen by removing a randomly chosen set of objects from the original object set. Those reducts frequently occurring in random subtables can be considered to be stable; it is these reducts that are encompassed by dynamic reducts (Bazan et al, 1994) [5]. Let $S=(U, C \cup D)$, where $\mathrm{U}$ is a non-empty, finite set called the universal, $\mathrm{C}$ is collection attributes set, $\mathrm{D}$ is decision attributes set, $B=\left(U^{\prime}, C \cup D\right), U^{\prime} \subseteq U$, B is called sub-decision system of S. Let $\rho(S)$ denote the set of all sub-decision system of $\mathrm{S}, F \in \rho(S)$ is called a $\mathrm{F}$ family of decision system $\mathrm{S}$. By $D R(S, F)$ we denote the set

$$
\operatorname{RED}(\mathrm{S}) \cap \bigcap_{\mathrm{B} \in \mathrm{F}} \operatorname{RED}(\mathrm{F})
$$

Any element of $D R(S, F)$ is called an F-dynamic reduct of $\mathrm{S}$. From the definition of dynamic reducts it follows that a relative reduct of $\mathrm{S}$ is dynamic if it is also a reduct of all sub table from a given family $\mathrm{F}$. This notion can be sometimes too much restrictive so, also a more general notion of dynamic reducts is applied. They are called $(F-\lambda)-$ Dynamic reducts, where $\lambda>0$. The set $D R(S, F)$, of all $(F-\lambda)$ - Dynamic reducts, is defined by

$$
\begin{gathered}
\mathrm{DR}_{\lambda}(\mathrm{S}, \mathrm{F})=\left\{\mathrm{Q} \in \operatorname{RED}(\mathrm{S}): \mathrm{SC}_{\mathrm{F}}(\mathrm{Q}) \geq \lambda\right\} \\
\mathrm{SC}_{\mathrm{F}}(\mathrm{Q})=\frac{|\mathrm{B} \in \mathrm{F}: \mathrm{Q} \in \mathrm{RED}(\mathrm{B})|}{|\mathrm{F}|}
\end{gathered}
$$

where is the F-stability coefficient of C. This lessens the 
previous restriction that a dynamic reduct must appear in every generated subtable. Now, a reduct is considered to be dynamic if it appears in a certain proportion of subtables, determined by the value $\lambda$.

For example, by setting $\lambda$ to 0.5 a reduct is considered to be dynamic if it appears in a least half of the subtitles. Note that if $F=\{S\}$, Then $D R(S, F)=R E D(S, F)$.

\section{DEFINITION OF GENERALIZED DyNAMIC REDUCTION}

According to the definition of dynamic reduction, if some feature attributes of any sub-decision system in F family are comprised by dynamic reduct, then it is certainly a feature attribute of decision system $\mathrm{S}$. This notion can be sometimes not convenient because the interest is in useful sets of attributes which are not necessarily reducts of the decision system [6], [7]. Therefore the notion of a dynamic reduct has to be generalized.

Definition 1 Decision system $S=(U, C \cup D)$, where $\mathrm{U}$ is a non-empty, finite set called the universal, $\mathrm{C}$ is condition attributes set, $\mathrm{D}$ is decision attributes $\mathrm{setF} \subseteq \mathrm{P}(\mathrm{S})$ then

$$
\bigcap_{\mathrm{B} \in \mathrm{F}} \operatorname{RED}(\mathrm{S})
$$

$G D R(S, F)$ is called the generalized dynamic reduct of decision system $\mathrm{S}$. From the definition of

$$
\mathrm{GDR}_{\lambda}(\mathrm{S}, \mathrm{F})=\{\mathrm{Q} \subseteq \mathrm{C} \mid \mathrm{SCF} \geq \lambda\}
$$

Generalized dynamic reducts it follows that any subset of S is a generalized dynamic reduct if it is also a reduct of all subtables from a given family $\mathrm{F}$. This notion can be sometimes too much restrictive so more general of generalized dynamic reducts is applied.

Definition 2 Decision system $S=(U, C \cup D)$, where $\mathrm{U}$ is a non-empty, finite set called the universal, $\mathrm{C}$ is condition attributes set, $D$ is decision attributes set and $F \subseteq P(S)$ and $\lambda \in(0.5,1]$, then the $\operatorname{GDR}_{\lambda}(\mathrm{S}, \mathrm{F})$ is called the $(\mathrm{F}-\lambda)$ generalized dynamic reduct of decision system $\mathrm{S}$.

If $Q \in R E D$ ( $B$ ) (for any $B \in F$ ) Then the number S CF is called the stability coefficient of the generalized Dynamic Reduct Q (relative F).

Proposition 2:
(1) $D R(S, F) \subseteq G D R(S, F)$
(2) $D R_{\lambda}(S, F) \subseteq G D R_{\lambda}(S, F)$ for any $\lambda \in(0.5,1]$
(3) If $S \in F$, then $D R(S, F)=G D R(S, F)$

\section{Statistical Arguments Showing the Introduced STABILITY COEFFICIENT OF DYNAMIC REDACTS}

Statistics is the science of making effective use of numerical data relating to groups of individuals or experiments. It deals with all aspects of this, including not only the collection, analysis and interpretation of such data, but also the planning of the collection of data, in terms of the design of surveys and experiments [8]. The data in most statistical problems relate to a sample drawn from some parent population or universe (as it is called in rough set theory). to calculate the set of decision rules based on a given decision table, a rules should be constructed which will proper not only for objects from this decision table but also for still not known example of objects (behind a given decision table). The dynamic reducts (defined in the previous sections) are calculated on family $\mathrm{F}$ of subtable created by random samples of a given decision table. The family $\mathrm{F}$ is of course only subfamily of all subtables the hypothetical universal decision table (including known and unknown object describing an actual considered aspect of reality). The family of all subtables of the universal decision table is denoted by $G$. the interest is in reducts which are the most frequently appearing in family $\mathrm{G}$, because the decision rules generated from this reducts are expected to be butter predisposed to designate value of the decision for objects from the whole universal decision table W. In another words the interest in the probability of the event, that a given $\mathrm{F}$ generalized dynamic reduct $\mathrm{R}$ is the reduct for all subtables from $\mathrm{G}$. This probability is denoted by and defined as the quotient $|\mathrm{GR}| /|\mathrm{G}|$ where: $P G(R)$

$$
\mathrm{GR}=\{\mathrm{B} \in \mathrm{G}: \mathrm{R} \in \mathrm{RED}(\mathrm{B}, \mathrm{D})\}
$$

We would like to define the F-dynamic reduct $\mathrm{R}$ for which the probability $P G(R)$ is not less than probability $P G$ for another dynamic reducts. However we cannot calculate $P G$ F-generalized dynamic reduct $\mathrm{R}$ because we do not know the whole family $G$. In this section we will see the so-called stability coefficient of any ( $F-\lambda)$-generalized dynamic reducts $\mathrm{R}$ is a proper measure of the probability. Then the stability coefficient of the $(F-\lambda)$-generalized dynamic reduct $R$ of decision table $\mathrm{S}$ is the maximum likelihood estimator of the probability $P G(R)$. The maximum likelihood estimator of the probability $P G(R)$ we denote by $M L E(P G(R))$.

Let us introduce the simple binomial distribution

$$
\mathrm{X}_{\mathrm{G}}^{\mathrm{R}}(\mathrm{B}) \rightarrow\{0,1\} \text { (For the family } \mathrm{G} \text { and reduct } \mathrm{R} \text { ) }
$$

Defined for any $B \in G$ :

$$
\begin{aligned}
& \mathrm{X}_{\mathrm{G}}^{\mathrm{R}}(\mathrm{B})=\left\{\begin{array}{l}
1: \mathrm{R} \in \operatorname{RED}(\mathrm{B}, \mathrm{D}) \\
0: \mathrm{R} \notin \operatorname{RED}(\mathrm{B}, \mathrm{D})
\end{array}\right. \\
& \text { Let } G 1=\left\{B \in G: X_{G}^{R}(B)=1\right\}
\end{aligned}
$$

Now it is easy to observe t.h at the probability $\mathrm{P}$ of the success in our binomial distribution is:

$$
\mathrm{p}\left[\mathrm{X}_{\mathrm{G}}^{\mathrm{R}}(\mathrm{B})=1\right]=\mathrm{P}_{\mathrm{G}}(\mathrm{R})
$$

and the probability of the defeat in our binomial distribution is:

$$
\mathrm{p}\left[\mathrm{X}_{\mathrm{G}}^{\mathrm{R}}(\mathrm{B})=0\right]=1-\mathrm{P}_{\mathrm{G}}(\mathrm{R})
$$

The probability of a success in $X_{G}^{R}$ distribution may be estimated by taking a sample of sub tables from the family $G$ next using the method of maximum likelihood estimator. The maximum likelihood estimator of success probability in our distribution is the arithmetic mean of values for all sub tables from the sample. 
Hence:

$$
\begin{aligned}
\operatorname{MLE}\left(\mathrm{P}_{\mathrm{G}}(\mathrm{R})\right)=\frac{\sum_{\mathrm{B} \in \mathrm{F}} \mathrm{X}_{\mathrm{G}}^{\mathrm{R}}(\mathrm{B})}{|\mathrm{F}|} \\
=\frac{\sum_{\mathrm{B} \in \mathrm{F} \cap \mathrm{G}^{1}} \mathrm{X}_{\mathrm{G}}^{\mathrm{R}}(\mathrm{B})+\sum_{\mathrm{B} \in \mathrm{F} \cap \mathrm{G}^{1}} \mathrm{X}_{\mathrm{G}}^{\mathrm{R}}(\mathrm{B})}{|\mathrm{F}|}
\end{aligned}
$$

From definition of $(F-\lambda)$-generalized dynamic reducts is concluded that the number $/ F \cap G / / F /$ sequel to the stability coefficient of the generalized dynamic reducts $\mathrm{R}$.

Theorem (1). The dynamic reducts with greater stability coefficient are "good" candidates for decision rules generation. They allow constructing rules with better classification quality of unseen object than reducts with smaller stability coefficients.

Theorem (2). Any dynamic reduct $\mathrm{R}$ the problem of calculating of $\operatorname{MLE}(P G(R))$ is equivalent to the problem of calculating of MLE of unknown success probability in binomial distribution [9]. From the moivre-laplace [11] theorem is obtained that the value $\operatorname{MLE}(P G(R))$ can be approximated for sufficiently large $/ F /$ (e.g. more than 150 subtables) by the normal distribution:

$$
\mathrm{N}\left(\mu, \sqrt{\frac{\mu(1-\mu)}{|\mathrm{F}|}}\right)
$$

where $\mu=|F \cap G|$ The minimal size of family $F$ (necessary to calculate generalized dynamic reduct can be estimated by the necessary sample size in case of normal distribution some assumptions about a confidence coefficient: 1- $\alpha$ (correctness of probability estimation) and maximal acceptable estimation error of $M L E(P G(R))$ (denoted by $\triangle M L E(P G(R))$ are to be made. The necessary size of family $F$ has to less than,

$$
\frac{\mathrm{t}_{\mathrm{a}}}{4 \Delta \mathrm{MLE}\left(\mathrm{P}_{\mathrm{G}}(\mathrm{R})\right.}
$$

where the number $t_{\alpha}$ is satisfying the equation:

$$
1-\alpha-\sqrt{\frac{2}{\pi}} \int_{0}^{\alpha} \exp \left(-\frac{\mathrm{t}^{2}}{2}\right) \mathrm{dt}
$$

Given the value of $t_{\alpha}$ one can read from the table of standardized normal distribution function. From figure 1, the $\operatorname{MLE}(P G(R)) \geq \lambda>0.5$ is

$$
\operatorname{MLE}\left(\mathrm{P}_{\mathrm{G}}(\mathrm{R})\right)\left(1-\operatorname{MLE}\left(\mathrm{P}_{\mathrm{G}}(\mathrm{R})\right)\right)
$$

Is decreasing there are

$$
\lambda(1-\lambda) \geq M L E\left(P_{G}(R)\right) .\left(1-M L E\left(P_{G}(R)\right)\right)
$$

as a dynamic in the case of reduct using $\lambda$ to represent $\operatorname{MLE}(P G(R))$, available:

$$
\frac{t_{a}^{2} / 2_{2} \cdot \lambda(1-\lambda)}{\left(\Delta \operatorname{MLE}\left(\mathrm{P}_{\mathrm{G}}(\mathrm{R})\right)\right)^{2}} \geq \frac{\mathrm{t}_{\mathrm{a}}^{2} / \mathrm{2}^{2} \cdot \mathrm{MLE}\left(\mathrm{P}_{\mathrm{G}}(\mathrm{R})\right)\left(1-\mathrm{MLE}\left(\mathrm{P}_{\mathrm{G}}(\mathrm{R})\right)\right.}{\left(\Delta \operatorname{MLE}\left(\mathrm{P}_{\mathrm{G}}(\mathrm{R})\right)\right)^{2}}
$$

For a given $\lambda$ value, will be a corresponding $|\mathrm{F}|$, expressed in the Man- $\mathrm{R}$ foot reduction coefficient $\lambda$ under the conditions of accuracy and objective sampling rate and therefore would be preferable:

$$
|\mathrm{F}| \geq \frac{\mathrm{t}_{\mathrm{a}}^{2} / \mathrm{2}_{2} \cdot \lambda(1-\lambda)}{\left(\Delta \mathrm{MLE}\left(\mathrm{P}_{\mathrm{G}}(\mathrm{R})\right)\right)^{2}}
$$

For Example: $\alpha / 2=0.05 \Rightarrow \alpha=0.1 \Rightarrow 1-\alpha=0.9$ Set $\triangle M L E(P G(R))=0.05$, there are

$$
\Phi\left(t_{a} / 2\right)-\left(t_{a} / 2\right)=0.9
$$

Namely $\Phi\left(t_{a} / 2\right)=(1+0.9) / 2$, through the standard normal distribution tables found in ta $/ 2=1.64$, are:

$$
|\mathrm{F}| \geq \frac{\mathrm{t}_{\mathrm{a}}^{2} / 2_{2} \cdot \lambda(1-\lambda)}{\left(\Delta \mathrm{MLE}\left(\mathrm{P}_{\mathrm{G}}(\mathrm{R})\right)\right)^{2}}=\frac{1.64^{2} \lambda(1-\lambda)}{0.05^{2}}=1076 \lambda(1-\lambda)
$$

The accuracy obtained reduction coefficient $\lambda=0.5$, then the $|F| \geq 269, \lambda=0.8$, then the $|F| \geq 173$. Then the confidence level, error estimation and stability coefficient can be the root of the values is according to the actual situation to decide, thus $|\mathrm{F}|$ value also has practical significance. Confidence level according to the $3 \sigma$ rule to calculate, there are $\mathrm{t} \mathrm{a} / 2=3 \alpha$, calculate a look-up table1. $\alpha=0.9973$, set $\triangle M L E(P G(R))=$ 0.05 , are:

$$
|F| \geq \frac{t_{a}^{2} / 2_{2} \cdot \lambda(1-\lambda)}{\left(\Delta \operatorname{MLE}\left(P_{G}(R)\right)\right)^{2}}=\frac{3^{2} \lambda(1-\lambda)}{0.05^{2}}
$$

IF $\lambda=0.5$, then the $|F| \geq 900, \lambda=0.8$ then the $|F| \geq 576$.These $F$ Values indicate a more comprehensive under the different accuracy of the dynamic reduct the sample analysis results, but the total calculation is very large. From the above, the value of $F$ is related to the confidence level 1- $\alpha$ and maximum likelihood estimate error $\triangle \mathrm{MLE}\left(\mathrm{P}_{\mathrm{G}}\left(\mathrm{R}_{\mathrm{i}}\right)\right)$. If the value of 1- $\alpha$, and $\Delta \operatorname{MLE}\left(\mathrm{P}_{\mathrm{G}}\left(\mathrm{R}_{\mathrm{i}}\right)\right)$ are changed, then the value of $|F|$ will change. Therefore, the value of, which Bazan have got, is according to a given level of confidence and a stable coefficient of errors. It cannot be obtained on condition of only setting the initial decision table. After introducing the accuracy factor of reduction, having considered the effect $\lambda$ had on the value of $F$, so $F$ can reflect the multi-level concept of dynamic reduction better, and in the premise that the confidence level and the error are acceptable, the problem can be simplified by means of considering $|\mathrm{F}|$ as a function of $\lambda$.

\section{IMPLEMENTATION STABILITY COEFFICIENT}

In this section offer a method for computing of dynamic reducts and reduct domain is presented. In this method a random set of subtitles from a given data table is taken and reducts for all these tables are calculated. In the first step some samples of the decision table are computed.

\section{EXPERIMENTAL RESULTS AND DISCUSSION}

Some tests were performed on the following data: market data [9], handwritten digits [7], texture recognition [6] and medical data [5].

For example:

30 samples with the size $90 \%$ of the decision table,

30 samples with the size $80 \%$ of the decision table, 
30 samples with the size $70 \%$ of the decision table, 30 samples with the size $60 \%$ of the decision table, 30 samples with the size $50 \%$ of the decision table,

Thus 150 new decision tables are received. The number of samples can be selected by taken into account the minimal family size calculated in the previous section. Next, reducts for all this tables are calculated. In the second step reducts with the stability coefficient higher than a fixed threshold are extracted. The reducts distinguished in such a way are treated as the true dynamic reducts.

\section{CONCLUSION}

Dynamic selections of attributes. Methods based on dynamic reducts or generalized dynamic reducts is used. The quality of unseen object classification based on in these dynamic reducts is much better than on the while set of attributes especially when data are noisy. The stability coefficient of a dynamic reduct is a very important because is equal to maximum likelihood estimator of probability that the dynamic reducts is a reduct of any subtables from a given decision table. The dynamic reduct are better predisposed classify new unseen objects than randomly chosen reduct.

Dynamic core consists with the most stable attributes which cannot be reducted, and describes the set of feature attributes. In these research dynamic reductions methods are in depth and extensively studied to discuss a number of levels of formal dynamic Simplified is about thinking, and gain a further description of the discussion following its formal foundation laid.

\section{REFERENCES}

[1] A. Skowron, "Boolean reasoning for decision rules generation," In: J. Komorowski, Z. W. Ras (eds.), in Proceeding of the Seventh International Symposium on Methodologies for Intelligent systems (ISMIS'93), Trondheim, Norway, pp. 295-305, 1993.

[2] J. Bazan, A. Skowron, and P. Synak, "Market data analysis: A rough set Approach," ICS Research Report 6/94 Warsaw University of Technology 1994c. J. Clerk Maxwell, a Treatise on Electricity and Magnetism, 3rd ed., vol. 2. Oxford: Clarendon, 1892, pp.68-73.

[3] S. R. Hesketh, A. Skrondal, and A. Pickles, "Maximum likelihood estimation of limited and discrete dependent variable models with nested random effects," Journal of Econometrics, 2005, pp. 301-323.

[4] Z. Pawlak and A. Skowron, "Rough sets and Boolean reasoning," Information Sciences, vol. 177, pp. 41-73, 2006.

[5] J. Bazan, A. Skowron, and P. Synak, " Dynamic reducts as a tool for extracting laws from decision tables, proceeding of the Eighth International Symposium on Methodologies for Intelligent systems (ISMIS'94)," Lecture Notes in artificial Intelligence, pp. 346-355, Berlin: Springer-verlag, 1994.

[6] T. Nguyen, R. Swiniarski, A. Skowron, and J. Baza, and K. Thyagarajan, "Application of Rough Sets, Neural Networks and Maximum likelihood for Texture Classification Based on Singular Value Decomposition," in Proceedings of the Third International Workshop on Rough Sets and Soft Computing, pp. 332-339. San Jose, California. 1994

[7] T. Nguyen, N. D. T. Son, and N. Hung, "An Approach to the Handwriting Digit Recognition Problem Based on Modal Logic," presented at ICS Research Report 44/93, Warsaw University of Technology, M.Sc. Thesis, Warsaw University, 1993.

[8] Z. Pawlak, "Rough Sets: Theoretical Aspects of Reasoning about Data," Kluwer, Dordrecht, 1991.

[9] W. Ziarko, "Rough Sets, Fuzzy Sets and Knowledge Discovery," Workshops in Computing, Springer Verlag and British Computer Society, 1994.

[10] A. Skowron and C. Rauszer, "The discernibility matrices and functions in information systems," In: $R$. S.owiński (ed.): Intelligent Decision Support. Handbook of Applications and Advances of the Rough Sets Theory, pp. 331 - 362, Kluwer, Dordrecht, 1992

[11] R. Podraza and W. Podraza, "Rough Set System with Data Elimination," in Proceedings of the 2002 International Conference on Mathematics and Engineering Techniques in Medicine and Biological Sciences (METMBS'2002), Las Vegas, Nevada, USA, 493-499, 2002. 